\title{
Experimental and Numerical Determination of Apparent Mass Variation of Granular Media Confined in Silo Geometry
}

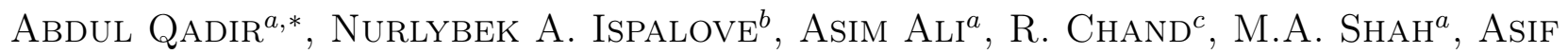 \\ $\mathrm{KHAN}^{a}$ AND KASHIF HUSSAIN ${ }^{a}$ \\ ${ }^{a}$ Department of Electrical Engineering, Sukkur Institute of Business Administration, Pakistan \\ ${ }^{b}$ S. Toraygyrov Pavlodar State University, Pavlodar 140008, Kazakhstan \\ ${ }^{c}$ Department of Physics, Faculty of Science, University of Malaya, Kuala Lumpur 50603, Malaysia
}

(Received April 27, 2015; in final form January 31, 2016)

\begin{abstract}
Granular materials have vast applications both in industry and in daily life. They display quite interesting and exceptional properties different from the other known forms of matter. To investigate the complex properties of particulate materials, experimental, analytical, and numerical techniques have been employed. In this paper the results of experimental and numerical tests of various grain sizes and coefficient of friction between granules and cylindrical walls on the mass measured at bottom of container, known as apparent mass, are reported. It is revealed that apparent mass augments with the grain size. Moreover, it is also found that the variation in apparent mass measurement is strongly dependent on bead diameter rather than the silo size. The results suggest that the conversion of vertical stresses into horizontal in silo is mainly due to the friction between the grain and system boundary than the arching phenomenon.
\end{abstract}

DOI: $10.12693 /$ APhysPolA.129.378

PACS/topics: 83.80.Fg, 45.70.Cc, 45.70.-n

\section{Introduction}

Granular packing has long been of interest to engineering and scientific community [1]. Since granular materials are ubiquitous, therefore any increased understanding can lead to numerous benefits $[2,3]$. Granular materials are simple to describe but they exhibit some non counterintuitive phenomenon for example, as in the case of sand pile where the maximum pressure is not found at the apex of pile, also in case of silo the pressure saturates and additional load is carried to side walls. This results in problems at design stage of silos that leads to expected silo failure. Nowadays silo geometry has emerged as an important tool to study the behaviour of granular media. By studying the form of force measured at the bottom much can be inferred about the stress distribution and propagation in granular systems.

The pioneering work on silo geometry was carried out by Janssen, it was demonstrated that mass measured at the base of a granular pile, known as apparent mass, does not vary linearly with the height of column, rather it saturates exponentially [4]. This is in contrast with the case of hydrostatics. Vanel et al. devised an experimental setup with an aim to measure the pressure profile of a slowly descending granular pile [5]. Furthermore, their investigations exhibited some differences between their experiments and the Janssen model. However, subsequent studies have shown that the Janssen model gives more accurate results compared with other

*corresponding author; e-mail: aqadir@iba-suk.edu.pk silo models [5]. Extensive studies have been carried out regarding the Janssen model however, few of them focuses the dependence of apparent mass measurement on the friction between silo wall and confined materials, due to inherent experimental difficulties.

In order to circumvent this problem computer simulation has appeared as an important tool. Using simulation techniques it has become easier to measure the parameters which are difficult to be investigate experimentally. Numerous methods have been evolved to investigate granular media in the two-dimensional systems. However, the prediction of these models and the approach to take the accurate simulation differs $[6,7]$. In threedimensional (3D) simulation usually the discrete element methods is employed.

In this paper using experimental setup and large-scale discrete element method (DEM) simulations in $3 \mathrm{D}$ the dependence apparent mass and coefficient of friction between silo walls have been investigated. The apparent mass is measured systemically by altering the friction values. It is found that apparent mass variation is also strongly dependent on coefficient of static friction between beads and silo wall, rather than the arching phenomenon only as usually believed [8].

\section{Experimental setup}

Some experimental tests have been carried out to provide the validation for simulation results. The experimental setup is shown in Fig. 1. The grains of mass $M$ are uniformly poured into the container by funnel placed on top. The bottom part of hollow cylinder is formed by a cylindrical piston. The piston alignment ensures 
that no leakage of grain takes place and also it does not touch the silo during experiment. Before each experimental run piston is inserted inside a vertical cylinder to a certain height, after the grains are filled a brief relaxation time (normally 30 to $60 \mathrm{~s}$ ) is allowed so that elastic energy accumulated by pressure sensor may be relaxed. Then the piston is allowed to descend at a slow velocity $(0.02 \mathrm{~mm} / \mathrm{s})$ on a total distance of $20 \mathrm{~mm}$. The moving speed of the piston is controlled by high performance electric motor. The aim of choosing the slow and longer downward motion of the piston is that to fully mobilize the friction force between the granules and the confining walls of cylinder. The bottom end of piston screwed onto the sensor and the force exerted by grains on the top horizontal surface of sensor is measured and a computer records data throughout the process. The corresponding force exerted by beads on the sensor is referred to as instantaneous apparent mass $M_{t}$, since its value is different from the total filled mass $M_{f}$.

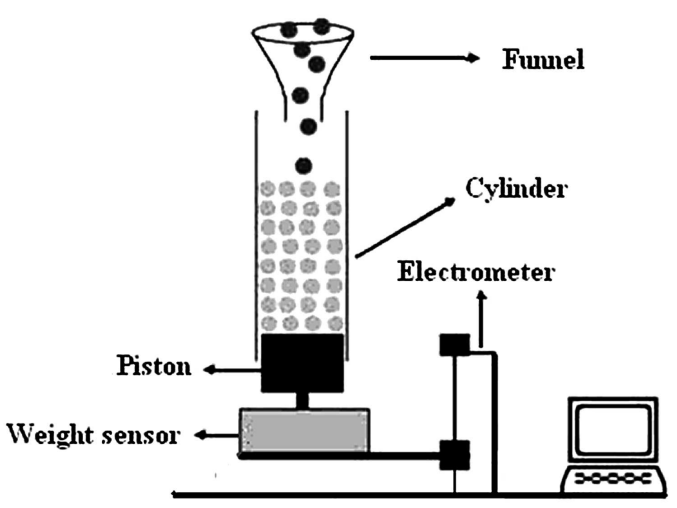

Fig. 1. The experimental setup.

\subsection{Measuring procedure}

Figure 2 displays the typical plot of instantaneous apparent mass $M_{t}$ as a function of filling mass $M_{f}$ of $600 \mathrm{~g}$ for beads diameter $d=4 \mathrm{~mm}$. It can be seen that the static pressure measured by the sensor exhibits comparatively larger fluctuations, in the beginning of descent of piston and eventually a relatively steady state is reached with smaller fluctuations. Here, we take the statistical average of 500 data points in the sequence of time series as depicted in the inset of Fig. 2, as a measuring value of apparent mass $M_{a}$. To assure the reproducibility and also reduce the error, the experiment is done 5 times, and then the average value of them is taken as a data point $M_{a}$.

Once the method of apparent mass measurement is defined, we vary the bead diameter $d=3,4,6,7,8$ and $12 \mathrm{~mm}$ while using the same size of silo $D=46.3 \mathrm{~mm}$. In such a configuration the variation of apparent mass on the filling mass is depicted in Fig. 3. The scatters are experimental results, and the lines represent a fitting from all data with different diameters of granules. It can

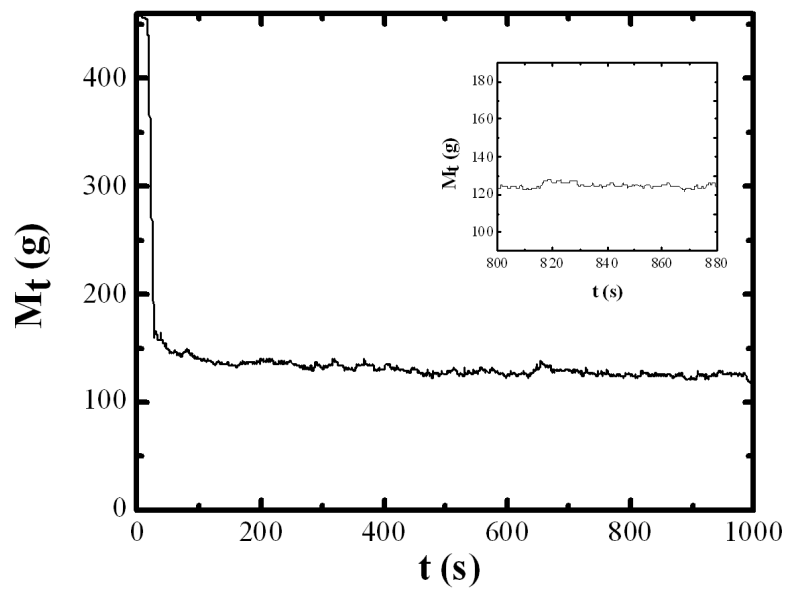

Fig. 2. Time variation of the instantaneous apparent mass. The filling mass of $600 \mathrm{~g}$ for $4 \mathrm{~mm}$ beads.

be observed that for low values of filling mass, $M_{a}$ is approximately equal to $M_{f}$ implying that in this region the hydrostatic behavior is more pronounced. For higher values of filling mass screening effect is dominant and $M_{a}$ illustrates a saturated state and the state has some fluctuations. The trend of every stress saturation curves is almost the same.

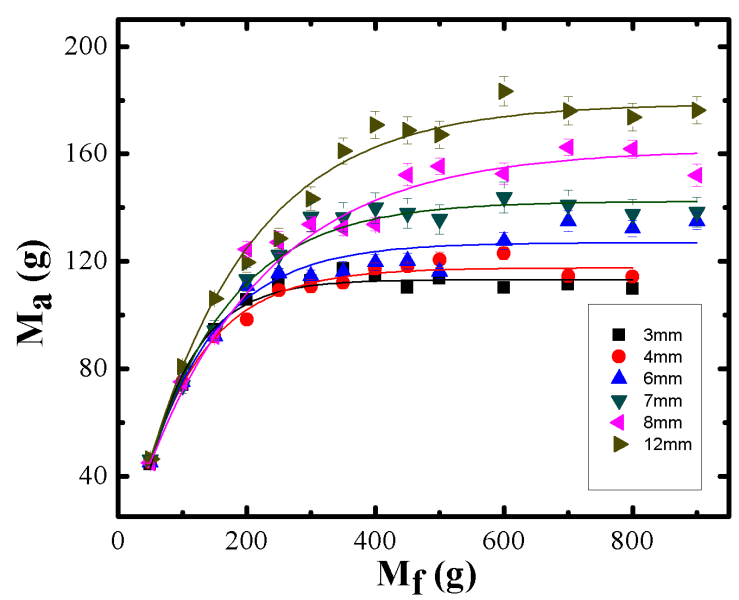

Fig. 3. Apparent mass as a function of filling mass for various grain sizes.

\section{Simulation method}

The DEM is a popular simulation technique in the study of granular media which is based on micro structural approach. It is employed here because it is difficult to measure experimentally the variation of apparent mass due to friction between beads and silo wall. This method was originally developed by Cundal and Strack with an aim to investigate the mechanical behavior of spheres and disks [6]. In this paper the simulation rests on the 
DEM code "LIGGGHTS" initially employed by Kloss et al. [7, 9]. The code is enhanced edition of the granular package of classical MD simulator LAMMPS [10] and is executed in a new Hertz-Mindlin granular contact model. The model assumes that the interactions among beads and confining boundary are like spring-dashpot in the perpendicular and parallel directions to their lines of centers.

For two adjacent beads $i$ and $j$ represented by position vectors $r_{i}$ and $r_{j}$ experience a particle overlap, given by $\delta=\left|r_{i j}-d\right|$, where $r_{i j}=r_{i}-r_{j}$, the force so produced is given by

$$
F_{n m}=F_{n}+F_{t}
$$

the normal force is represented by $F_{n}$ :

$$
F_{n}=f(\delta / d)\left(k_{n} \delta n_{i j}-\frac{m}{2} \gamma_{n} v_{n}\right)
$$

and tangential force by $F_{t}$ :

$$
F_{t}=f(\delta / d)\left(-k_{t} \Delta s_{t}-\frac{m}{2} \gamma_{t} v_{t}\right),
$$

whereas $v_{n}$ and $v_{n}$ represent the relative surface velocity normal and tangential components, $m$ is the mass of particles and $\Delta s_{t}$ is elastic tangential displacement among grains. While $k_{n, t}$ and $\gamma_{n, t}$ are elastic and viscoelastic constants, respectively. For Hertzian contacts, $f(x)=\sqrt{x}$.

This paper presents two modes of simulations. In the first series of simulation, keeping the grain diameter constant i.e. $d=2.0$ units. While friction between the particle and system boundary $\mu$ has been varied as 0.20 , $0.40,0.60,0.80$ and 1.00 . The convenient time unit $\tau=\sqrt{d / g}$ is adopted. Here, time-step of $\delta t=10^{-4 \tau}$, $k_{n}=4 \times 10^{5} \mathrm{mg} / d$ and $k_{t}=2 / 7 k_{n}$ and damping coefficients $\gamma_{n}=\gamma_{t}=1000 \sqrt{d / g}$ are used. In the next series of simulation, keeping the friction coefficient $\mu$ constant the grains diameter is varied from $d=2.0$ to $4.0,5.0,6.0$. The whole simulation has been carried out with fixed set of parameters and measurement of quantities is in dimensionless units based on $m, g$, and $d$.

In these simulations, granular packing has been generated by the method of pouring $N$ mono-disperse beads from a fixed height of $z=280$ units. The height of granular column is set equivalent to 6 times the diameter of silo. The packing is allowed to rest under gravity. In the beginning volume fraction was found to be 0.13 and finally reaches $\approx 0.615$. The model and techniques can be found in contemporary research [11-13]. The simulations were run until the kinetic energy per bead became the least. It is achieved by descending the base of silo. Such packing, known as quiescent, has been adopted for further investigation. Pilings are examined before and after cessation of base movement.

Figure 4 shows the structure of a packing with base velocity $(0.001 \mathrm{~d} / \mathrm{g})$ in the downward direction $(-z)$. Then notable particle rearrangement takes place during the descending of pile. Many beads initially in touch with the confining boundary moved upward. Moving beads are coloured according their velocities in order to give a better visualization of beads movement as time elapses. During motion, the packing height does not change.

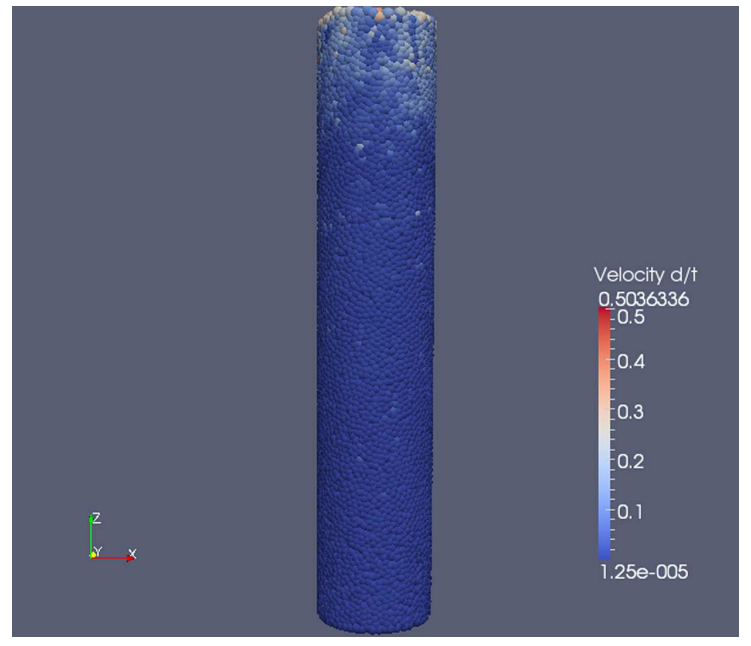

Fig. 4. Snapshot of simulation of granular packing.

\section{Results and discussion}

Figure 5 shows the variation in apparent mass measurement with the filling mass. It is revealed that for lower filling mass values the granular material exhibits behavior similar to the hydrostatic case. However, for higher filling mass values the apparent mass becomes constant and a saturated state is attained. It may be noted that stress curves augment and then asymptote. Moreover, the stress curves diverge with bead size.

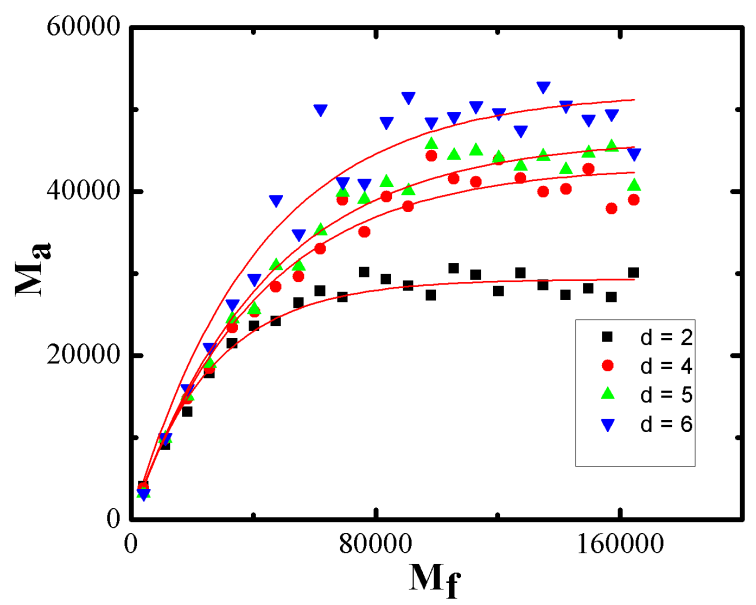

Fig. 5. Evolution of apparent mass with the filling mass.

This branching of data lines needed further investigation as bead size influence the stress transmission in a silo. The reason of such phenomenon lies in the Janssen model, used in silo design [4]. The Janssen model is a classical approach to describe the screening of vertical 
stresses in silos. The original papers date from 1895 and became very popular, certainly because the model could reproduce the correct phenomenology with an extremely simple mathematical framework. Janssen presented a theory regarding the stress distribution in a silo. The central observation is that stresses, measured at the bottom, are generally much smaller than the hydrostatic pressure $\rho g H$ which we would have in a liquid (here $\rho$ is the density, $g$ is the gravitational acceleration, and $H$ is the column height). It was assumed in the theory that: (a) The horizontal stresses in the granular medium are proportional the vertical stresses,

$$
\sigma_{x x}=K \sigma_{z z}
$$

where $K$ is redirection parameter. (b) Another simplification was made concerning friction. It was assumed that friction between the walls of silo and grains have reached at the maximum and are in yield criterion. (c) The density of material is also considered constant over all depths.

Considering a horizontal slice of height $\mathrm{d} h$ and radius $R$ the Janssen model assumes that the weight of beads in the slice is balanced by variation of pressure of the surrounding granules and the friction force between the granules and the confining walls of container, we get

$$
\frac{\pi R^{2} \mathrm{~d} \sigma_{z z}}{\mathrm{~d} h} \mathrm{~d} h+2 \pi R \mu K \sigma_{z z} \mathrm{~d} h=\pi R^{2} g \rho \mathrm{d} h .
$$

On simplification it reduces to

$$
\frac{\mathrm{d} \sigma_{z z}}{\mathrm{~d} h}+\frac{\sigma_{z z}}{\lambda}=\rho g \text {. }
$$

$\lambda=\frac{R}{2 \mu K}$ is a central parameter in the Janssen theory known as effective screening length it characterizes the height above which the weight is screened out by the boundary walls, whereas the coefficient $K$ describes the deflection of vertical stresses into horizontal ones.

Owing to screening effect the mean pressure at bottom is only a part of total filling mass known as effective mass since the rest is screened out by the container walls. The relationship between the effective mass and the saturation mass is as follows:

$$
M_{a}=M_{s}\left(1-\mathrm{e}^{-M_{f} / M_{s}}\right),
$$

where saturation mass

$$
M_{s}=\rho \pi(D / 2)^{3} /(2 \mu K) .
$$

$M_{f}$ is the filling mass, while material density is represented by $\rho$, and $\mu$ being the friction coefficient between grains and wall, the horizontal and vertical stresses ratio is denoted by $K$. Equation (3) does not provide any relation regarding the bead size and saturation mass. However, it can be used to extract the values of saturation mass by simulating the data point lines. The solid lines depicted in Fig. 5 are also obtained by using Eq. (3). It is illustrated that with the augment in grain diameter, the splitting of stress saturation curves occurs. The redirection of vertical stresses towards the confining boundary is the main reason for such variation.

From Fig. 5 the splitting of data lines with augment in $d$ may be due to the reason that conversion of vertical to horizontal stresses is reduced with the augment in bead size. It implies that when granular column comprises of smaller bead sizes there is strong tendency of conversion of vertical stress into the horizontal ones. Thus redirection parameter $K$ is efficient in such configurations. The reason lies in the fact that number of contact points between the beads and silo is reduced in the presence of larger size of grains. Hence more stress is propagated to the base of silo rather than to the side wall. Consequently the screening effect is minimized in the case of larger size of grains and higher saturation mass values are obtained. It appears that friction between grains and the silo wall leads to the variation in apparent mass.

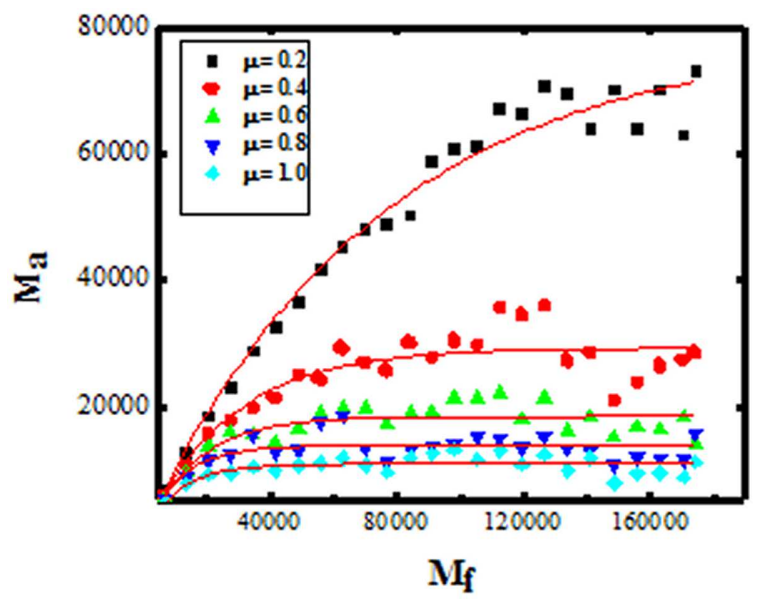

Fig. 6. Variation of apparent mass with filled mass for various coefficient of friction.

To further elaborate the role of friction in variation of apparent mass, the simulations are run for the same bead diameter while the friction coefficient between the grains and the silo wall has been varied. When the grain diameter is $d=2.0$ units the friction coefficient is changed from $\mu=0.20,0.40,0.60,0.80$ to 1.00 . It can be observed from Fig. 6 that augmentation of friction values results in minimizing the apparent mass. Figure 6 also shows that for $\mu=0.20$ the granular material exhibits hydrostatic behavior in a silo. The behavior does not alter even for different grain sizes. However in this paper results of bead diameter $d=2$ units are presented. Generally, it is assumed that screening effect in granular media is due to the arching [8]. However, the presented research using different values of friction between beads and container wall it is revealed that in addition to the arching, the friction also plays an important role in shielding of weight in silos.

\section{Conclusion}

Experimental tests and large-scale simulations have been carried out to measure the mean vertical pressure at the base of cylindrical granular pile. It is shown that the classical Janssen analysis predicts the saturation of 
mass in a granular column which is also observed in our simulation results. It is found that the shielding effect is a macroscopic one and it should not be attributed only to the arching, but also to the friction between grains and the confining wall of the silo. Furthermore, it has been observed that the stress transmission at the bottom of packing also depends more on the size of grains than the size of silo. In future this work will be corroborated with the role of number of contact point at different conditions on the apparent mass so as to further elaborate the screening effect. Thus, more analyses such as the averaged normal force and tangential force can be carried out.

\section{References}

[1] L.P. Kadanoff, Rev. Mod. Phys. 71, 435 (1999).

[2] J.S. Leszczynski, T. Blaszczyk, Granul. Matter 13, 429 (2011).

[3] T.S. Majumdar, R.P. Behringer, Nature 435, 1079 (2005).
[4] H. Janssen, A.Z. Vereins, Dtsch. Eng. 39, 1045 (1895).

[5] L. Vanel, Ph. Claudin, J.P. Bouchaud, M.E. Cates, E. Clément, J.P. Wittmer, Phys. Rev. Lett. 84, 1439 (2000).

[6] P.A. Cundall, O.D.L. Strack, Geotechnique 29, 47 (1979).

[7] C. Kloss, C. Goniva, A. Hager, S. Amberger, S. Pirker, Prog. Comput. Fluid Dyn. 2, 3 (2012).

[8] Anita Mehta, Granular Physics, 2011.

[9] C. Kloss, C. Goniva, in: Proc. Fifth Int. Conf. on Discrete Element Methods, London, 2010, p. 25.

[10] F. Bertrand, L.A. Leclaire, G. Levecque, Chem. Eng. Sci. 60, 2517 (2005).

[11] B. Gilles, E. Leveque, C. Laroche, C. Coste, Phys. Rev. Lett. 92, 20 (2004).

[12] J.W. Landry, G.S. Grest, L.E. Silbert, S.J. Plimpton, Phys. Rev. E 67, 4 (2003).

[13] J.W. Landry, G.S. Grest, S.J. Plimpton, Powder Technol. 139, 233 (2004). 